SHORT COMMUNICATION

\title{
Environmentally induced changes in carotenoid-based coloration of female lizards: a comment on Vercken et al.
}

\author{
J. COTE, ${ }^{\star}, \dagger$ J.-F. LE GALLIARD, ${ }^{*},+$ J.-M. ROSSI* \& P. S. FITZE§, \\ *Laboratoire Fonctionnement et Evolution des Systèmes Ecologiques, CNRS UMR 7625, Université Pierre et Marie Curie, Paris, France \\ $\dagger$ Department of Environmental Science and Policy, University of California, Davis, CA, USA \\ \$Centre de Recherche en Ecologie Expérimentale et Prédictive, Station biologique de Foljuif, Ecole Normale Supérieure, St Pierre les Nemours, France \\ $\S$ Department of Biodiversity and Evolutionary Biology, Museo Nacional de Ciencias Naturales (MNCN-CSIC), Madrid, Spain \\ -Instituto Pirenaico de Ecología (IPE-CSIC), Avenida de Galicia s/n, Jaca (Huesca), Spain
}

\section{Keywords:}

adult sex ratio;

coloration;

phenotypic plasticity;

polymorphism.

\begin{abstract}
Colouration may either reflect a discrete polymorphism potentially related to life-history strategies, a continuous signal related to individual quality or a combination of both. Recently, Vercken et al. [J. Evol. Biol. (2007) 221] proposed three discrete ventral colour morphs in female common lizards, Lacerta vivipara, and suggested that they reflect alternative reproductive strategies. Here, we provide a quantitative assessment of the phenotypic distribution and determinants of the proposed colour polymorphism. Based on reflectance spectra, we found no evidence for three distinct visual colour classes, but observed continuous variation in colour from pale yellow to orange. Based on a 2-year experiment, we also provide evidence for reversible colour plasticity in response to a manipulation of the adult population sex ratio; yet, a significant portion of the colour variation was invariant throughout an adult female's life. Our results are thus in agreement with continuous colour variation in adults determined by environmental factors and potentially also by genetic factors.
\end{abstract}

\section{Introduction}

Colour signals are widespread in animals that rely on vision for species recognition, intra-specific competition or mate choice (e.g. Endler, 1991; Galeotti et al., 2003). Colour variation consists either of graded variation (i.e. continuous variation) or of discrete developmental switches (i.e. discrete colour morphs) and is determined by the combined action of genetic and environmental factors (e.g. Hill \& Montgomerie, 1994; Hoffman \& Blouin, 2000; Roulin, 2004). Some species display discrete colour morphs that are mainly genetically determined; these morphs are associated with alternative behavioural strategies maintained by disruptive genetic selection (Thompson et al., 1993; Sinervo \& Lively,

Correspondence: Julien Cote, Department of Environmental Science and Policy, University of California, Davis, CA 95616, USA

Tel.: +1 530754 9307; fax: +1 530754 7383;

e-mail: jdcote@ucdavis.edu
1996). On the other hand, coloration is often a continuous trait whose expression changes with body condition, health status and environmental condition (e.g. Hill \& Montgomerie, 1994; Fitze et al., 2003). The evolution of condition-dependent coloration typically involves honest signalling caused by trade-offs between colour pigments, antioxidant capacities and immune defences (Olson \& Owens, 1998).

Genetic morph determination is more likely to evolve in species characterized by low migration, unreliable environmental cues and strong disruptive selection (Sinervo \& Calsbeek, 2006). Potential examples of discrete, genetic colour morphs have been described in birds (reviewed in Roulin, 2004), fishes (Roff, 1996), amphibians (Hoffman $\&$ Blouin, 2000) and lizards (reviewed in Cooper \& Greenberg, 1992). Colour variation is common in lizards where it involves various body parts and colour pigments, and can be associated with both intra-and inter-sexual interactions (Cooper \& Greenberg, 1992; LeBas \& Marshall, 2000; Weiss, 2006). To date, genetic 
colour morphs have been described convincingly in Uta stranburiana (Sinervo \& Lively, 1996) and Urosaurus ornatus (Thompson et al., 1993). A recent study hypothesized that female common lizards (Lacerta vivipara) exhibit three distinct visual colour classes (pale yellow, orange and intermediate) that represent alternative, genetically determined reproductive strategies (Vercken et al., 2007). However, as pointed out by the authors, the distribution of spectrophotometer measurements on females - a physical assessment of their reflectance properties (Endler, 1990) - appeared continuous among and within the colour classes (Vercken et al., 2007). Ventral colour in female common lizards may thus be a continuous trait which appears discrete to the human eye. Here, we provide a quantitative assessment of the frequency distribution and determinants of the colour variation previously reported in female L. vivipara by Vercken et al. (2007). Adult common lizards show sexually dimorphic coloration from the throat to the basis of the tail (Vercken et al., 2007). In our study populations, females are duller than males, their ventral coloration ranges from pale yellow to orange (Fig. 1) and stems from carotenoids but not from melanins or pteridins (extraction of skin pigments; (Cote, 2006). Throat and belly coloration is involved in communication, including mate recognition (Bauwens, 1987) and female-female competition (Vercken $\&$ Clobert, 2008). We tested the existence of a discrete colour polymorphism by estimating the distribution of quantitative colour parameters in adult females (hue, brightness and chroma, Endler, 1990) and the individual consistency of colour parameters across two field seasons. If female common lizards exhibit discrete colour classes distinct to the human eye, we should detect clusters of colour parameters and colour parameters should be highly consistent across study years.

Condition-dependent colour signals typically involve carotenoids, or pteridins, in fishes, birds and reptiles (Olson \& Owens, 1998; Weiss, 2006). Carotenoids cannot be synthesized de novo by lizards and thus must be acquired by the diet and allocated between colour traits and other functions (Olson $\&$ Owens, 1998). This creates trade-offs between carotenoid-based pigmentation and health components, including nutritional condition (Fitze et al., 2003), parasite exposure (Milinski \& Bakker, 1990) or immunocompetence (Møller et al., 2000). Thus, carotenoid-based ventral coloration might indicate individual quality in female common lizards, as well as the quality of the social environment. To test this hypothesis, we examined the relationship between colour parameters, body size and post-parturition body condition and we tested for the flexibility of colour parameters in response to a transient deterioration of the social environment. To do so, we enhanced the adult sex ratio (ASR) from a female-biased (typical of the mean ASR in the wild) to a male-biased sex ratio (typical of the upper range of ASR in the wild) during 1 year (Le Galliard et al., 2005). We next monitored the females for an additional year in a common garden with a female-biased ASR. Previous studies showed that male-biased ASR impacts
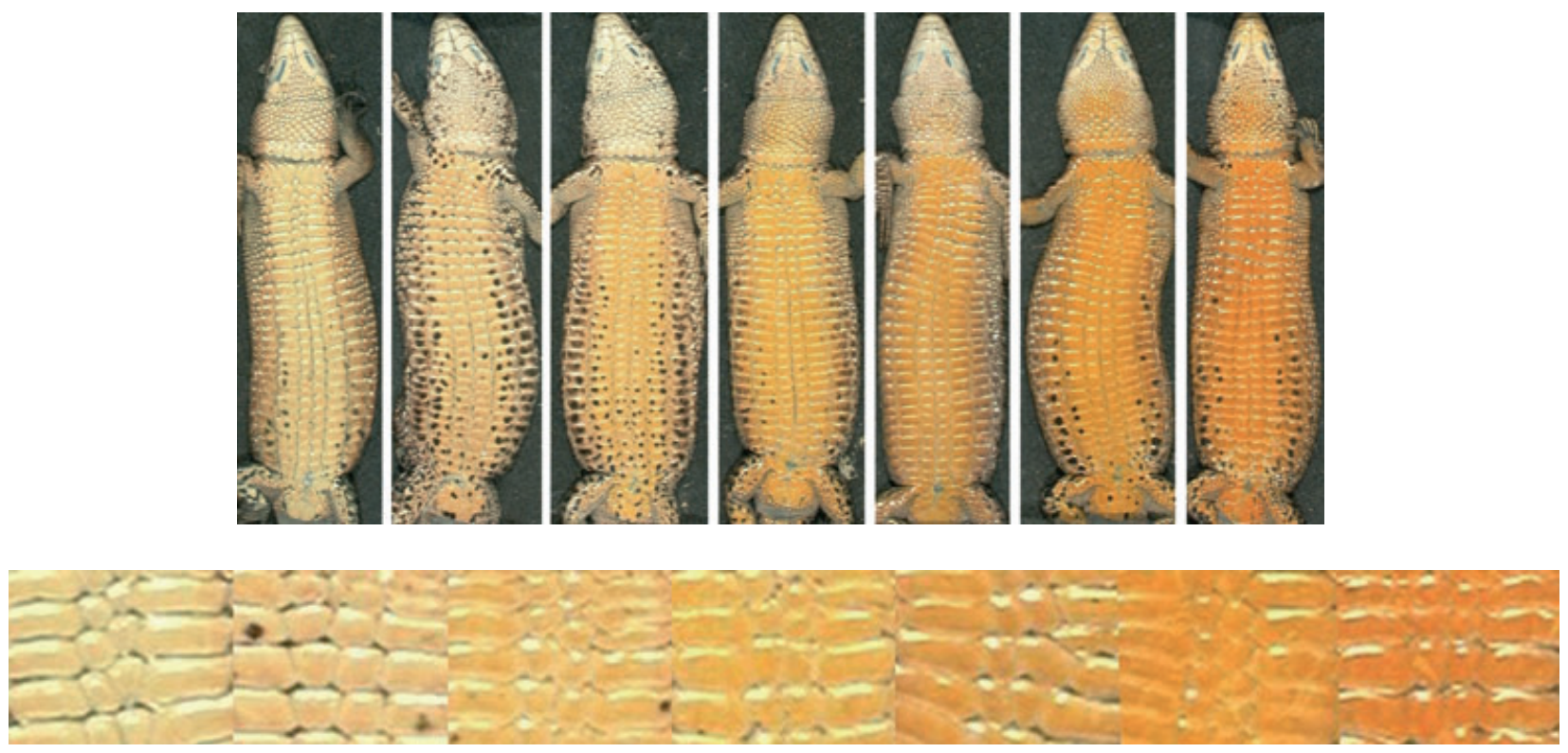

Fig. 1 Individual variation in ventral coloration in adult female common lizards Lacerta vivipara from the Cevennes area, southern France. Digital pictures were taken in standardized conditions. Photographs from the left to the right show a continuum from pale yellow to yellow, yellow-orange and orange ventral colouration. 
negatively the lifetime reproductive success of female common lizards due to higher male aggression during mating, involving direct physical effects, stress and loss of energy due to sexual harassment by males (Fitze et al., 2005). Here, we tested for a change in ventral coloration of females in response to male excess.

\section{Material and methods}

\section{Field experiment}

We maintained 12 experimental populations in enclosures located in a natural meadow at the Ecological Research Station of Foljuif (France, $60 \mathrm{~m}$ a.s.l., $48^{\circ} 17^{\prime} \mathrm{N}$, $\left.2^{\circ} 41^{\prime} \mathrm{E}\right)$. The enclosure's habitat matched the natural habitat of the species and each enclosure $\left(10 \times 10 \mathrm{~m}^{2}\right)$ was surrounded by plastic walls to prevent lizards from escaping. Lizards introduced in these populations at the start of the study (June 2002) were obtained from natural populations of the Cévennes area $\left(44^{\circ} 30^{\prime} \mathrm{N}\right.$, $3^{\circ} 45^{\prime} \mathrm{E}, 1400-1600 \mathrm{~m}$ a.s.l.) and marked by toe clipping. To enhance exposure to males, we created six populations with a male-biased ASR (14 adult males and four adult females) and compared the fate of females from these populations with that of females from six populations with a female-biased ASR (four adult males and 14 adult females). Yearling ( $n=12$ per population) and juvenile ( $n=42-45$ per population) sex ratios were held constant and balanced $(50: 50)$ in all populations.

Population structures and sex ratios correspond to range of variation in natural populations (Le Galliard et al., 2005). Natural range of population sex ratios was assessed with a data base of ASR for entire populations in France, Belgium and the Netherlands, and with measures of ASR in areas spanning the maximum size of female home ranges in a natural reference population. The population data base showed that ASR varied among populations and within populations in time from 0.15 to 0.65 (mean $0.39 \pm 0.12 \mathrm{SD}, n=33$ estimates from 14 populations). In the reference population, the estimated ASR inside neighbourhoods varied from 0 to 0.77 between years and neighbourhoods (mean $0.18 \pm 0.18$ SD, 22 neighbourhoods surveyed during 12 years). Furthermore, a proportion of approximately $10 \%$ of the neighbourhoods had ASR larger than 0.70 in the same reference population. Hence, the ASR of the femalebiased populations was similar to the mean ASR of the reference population, whereas the ASR of male-biased populations was within the $10 \%$ most male-biased ASRs. Therefore, the sex ratio treatments applied in the ASR experiment cover the range of sex ratio variations observed across and within populations of this species.

After 1 year of ASR manipulation, we recaptured all live females in June 2003 and kept them in individual cages in the laboratory until they laid their eggs. In JuneJuly 2003, we released all live females $(n=148)$ from male-biased $(\mathrm{MB})$ and female-biased (FB) populations
2-3 days after parturition into new populations with a female-biased ASR ( $36 \%$ of males, $n=28$ adults per population). Yearling ( $n=12$ per population) and juvenile ( $n=103 \pm 3.1$ SE per population) sex ratios were held constant and balanced (50:50) in all populations. Within each enclosure, the proportions of females originating from $\mathrm{MB}$ and $\mathrm{FB}$ populations were similar. We kept females in these enclosures during one additional year and recaptured all live lizards in each enclosure in June 2004. The experimental procedures has been described in greater detail elsewhere (Le Galliard et al., 2005).

\section{Colour measurements}

Visual inspection of lizards and standardized digital pictures show that individuals exhibit substantial variation in ventral coloration (see Fig. 1 and photo galleries available as Supplementary material). Exclusive of black spots and brownish pigmentation, ventral coloration in female common lizards ranges from pale yellow to bright orange. We first estimated coloration in all adult females using a colour reference with three distinct groups (pale yellow, bright orange and intermediate coloration) following the classification proposed by Vercken et al. (2007). Vercken et al. (2007, p. 223) defined the intermediate females as females with 'intense yellow' or 'mixture of yellow and orange'. We optically evaluated the importance of mixed coloration using digital photos (see Supplementary material). Importantly, this analysis demonstrates that colour heterogeneity between and within scales is rare and that colouration variance among scales is not significantly higher in intermediate females (see Supplementary material). These analyses therefore suggest the absence of a well-defined mixed yellow and orange colouration class in female common lizards from this natural range.

Second, we measured the ventral coloration of all adult females on the middle of the thorax before release (June 2002) and one day after each recapture (June 2003 and June 2004). We used a spectrophotometer (USB2000; Ocean Optics Inc., Dunedin, FL, USA), a xenon light source (PX-2; Ocean Optics Inc., measurement area of ca. $0.12 \mathrm{~cm}^{2}$ ) and a reflection probe (R400-7-UV/VIS; Ocean Optics Inc.). Measurements on the middle of the thorax were taken as they represent the coloration of the entire belly (J. Cote and P.S. Fitze, unpublished data, see also Fig. 1). Furthermore, measurements on the middle of the thorax correlate well with average colour of the belly assessed using digital photos (see Supplementary material). We derived brightness, hue (redness) and chroma (purity) of the ventral coloration from the reflectance spectrum, according to Endler's segment classification method (Endler, 1990) and following the procedures used in Vercken et al. (2007). Spectrophotometer measurements were significantly repeatable $(n=109$ adults measured on the thorax, three measurements per individual; chroma $F_{108,218}=5.17, \quad P<0.0001$, 
$r=0.58$; hue: $F_{108,218}=8.59, \quad P<0.0001, \quad r=0.72$; brightness: $\left.F_{108,218}=2.74, P<0.0001, r=0.37\right)$.

\section{Statistical analyses}

We conducted all statistical analyses using $\mathrm{R}$ 2.6.0 software (Free Software Foundation, GNU Project, Boston, MA, USA). We checked for the normality of spectrophotometer data in the initial sample to ascertain if coloration varied gradually or not. Using the same sample, we regressed each spectrophotometer measurement (chroma, hue and brightness) against female size (snout vent length, SVL), body condition (residuals of a linear regression of body mass against body size) using the Im procedure (Venable \& Ripley, 1999). We compared spectrophotometer measurements among visual colour classes of females using anovas. We also investigated whether discrete colour classes exist in the multidimensional spectrophotometer data. To do so, we used the model-based clustering method for normal mixtures of mclust, which allows fitting a covariance model and number of clusters using an expectation-maximization algorithm and Bayesian information criterion (BIC) model selection procedure (Fraley \& Raftery, 1999). The final model was selected among the 10 available default covariance models with a range of clusters chosen between one and five (for more details, see Fraley $\delta$ Raftery, 1999). The best model (highest BIC) was chosen to classify female colour measurements, to calculate classification uncertainty, and to compare the corresponding classification with the visual colour classes.

We next analysed the inter-annual changes in spectrophotometer measurements during and after the sex ratio manipulation using mixed-effect models in the lme procedure (Pinheiro \& Bates, 2000). We used repeated measurements of thorax coloration to fit statistical models including time (measurement year) as a fixed effect, ASR treatment and the interaction between ASR and time effects, as well as the random effect of female identity. We used only females for which two observations were available and our results are therefore not confounded by differential mortality between the treatments. The amount of variation observed between individual females was used to calculate the inter-annual consistency of colour parameters (intra-class correlation coefficient, ICC). This analysis produced similar results as the colour change analysis (difference in coloration between repeated measurements) where we included the random effect of enclosure identity nested within the ASR treatment. Consequently, we only present the analyses on the inter-annual consistency of colour parameters. Fixed effects were tested with conditional tests by F-tests based on the usual (REML) conditional estimate of the variance and random effects were tested with asymptotic likelihood ratio tests (Venable \& Ripley, 1999). (a)

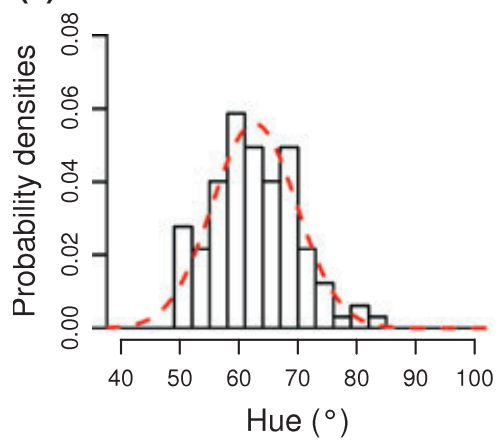

(c)

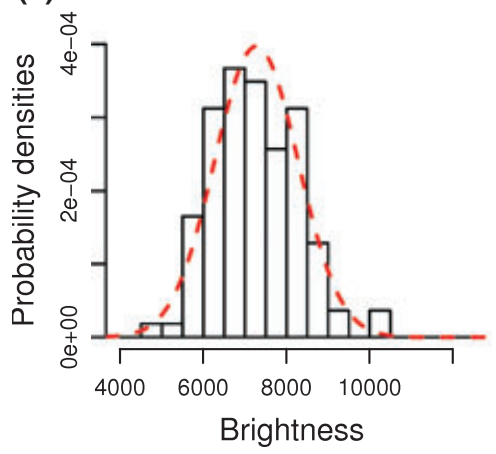

(b)

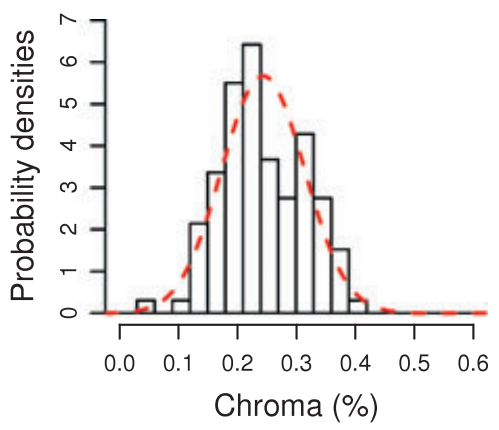

(d)

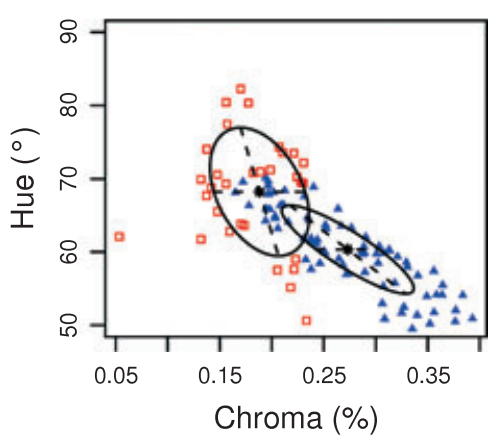

Fig. 2 Frequency distribution of spectrophotometer measurements on the thorax of adult female common lizards. $(\mathrm{a}-\mathrm{c})$ Univariate data for hue, chroma and brightness measured with a spectrophotometer on the thorax of adult females $(n=109)$ were obtained before the adult sex ratio manipulation. The normal distribution was plotted against the frequency distribution of each spectrophotometer parameter to allow visual comparison with the Gaussian distribution. There was one notch in the univariate data for hue and chroma, and the bivariate data indeed showed two clusters of females according to the normal mixture modelling (best model with one cluster: BIC -431.45 ; best model with two clusters: BIC -416.11 ; best model with three clusters: BIC -418.71 ; see Fraley \& Raftery, 1999 for more details). (d) Coordinate projections with classification and contour lines of the normal mixtures from the best model (VEV model, two clusters). The classification uncertainty was quite high (mean 0.17) given the overlapping distributions of the two normal mixtures. 


\section{Results}

The frequency distributions of spectrophotometer data matched normal distributions (Shapiro-Wilk normality tests, $n=109$; chroma: $W=0.983, \quad P=0.19$; hue: $W=0.982, P=0.14$, brightness: $W=0.985, P=0.26$, see Fig. 2). The variation in chroma and hue among females was best explained by female size rather than female condition (SVL for chroma: $F_{1,105}=4.25$, $P=0.04$; SVL for hue: $F_{1,105}=9.01, P=0.003$; condition for chroma: $F_{1,105}=1.89, P=0.17$; condition for hue: $\left.F_{1,105}=0.37, P=0.54\right)$. SVL and body condition were not correlated with brightness (all $P>0.12$ ). The purity (chroma; slope $0.0035 \pm 0.0016 \%$ per $\mathrm{mm}$ ) and the redness of the coloration (hue; slope $-0.573 \pm 0.189^{\circ}$ per $\mathrm{mm}$ ) increased with the body size of the female. The number of initially pale yellow, intermediate and orange females was 49, 51 and 9 respectively. There were strong, significant differences in chroma $\left(F_{2,106}=64.37\right.$, $P<0.0001)$, hue $\left(F_{2,106}=30.56, P<0.0001\right)$ and brightness between the visual colour classes $\left(F_{2,106}=16.06\right.$, $P<0.0001)$ as found in Vercken et al. (2007). Chroma increased and hue and brightness decreased from pale yellow to bright orange females. No cluster of individuals was found in the one-dimensional spectrophotometer data, but two clusters were found in the two-dimensional data of chroma and hue (Fig. 2). However, this clustering discriminated poorly the pale yellow females from the two other visual colour classes (cluster 1: 23 pale yellow, 45 intermediate and nine orange females; cluster 2: 26 pale yellow and six intermediate females). A similar model-based clustering based on the two-dimensional data of chroma and hue reported by Vercken et al. (2007) in Fig. 1b, which included a larger sample size directly captured in the field $(n=245)$, provided no evidence for clusters of spectrophotometer measurements (best model with one cluster: BIC -1072.72; best model with two clusters: BIC -1073.98; best model with three clusters: BIC -1077.65; see Fraley \& Raftery, 1999 for more details).

We recaptured 62 females and measured their thorax coloration at the end of the sex ratio manipulation. Analyses of repeated measurements indicated a significant effect of the ASR manipulation on inter-annual changes in hue and marginal ASR effects on inter-annual changes in chroma and brightness (see Table 1). In addition, we found significant inter-individual variation in coloration, and therefore inter-annual consistency in colour parameters (see ICCs in Table 1). During the course of the sex ratio experiment, the ventral coloration of females in $\mathrm{MB}$ populations got more pale (hue change $11.53 \pm 4.05 \mathrm{SE}$ ), whereas it did not change in females of FB populations (change $-1.83 \pm 1.53 \mathrm{SE}$, see also Fig. 3). Yet, no ASR effect on the changes in visual colour score defined by Vercken et al. (2007) was found (general linear mixed-effect model, $\left.F_{1,7}=0.57, P=0.47\right)$. The thorax coloration of 24 females could be measured a third time, 1 year after the end of the ASR manipulation (Table 1). We found a significant effect of the ASR manipulation on repeated measures of hue and consistent inter-individual variation for hue. Thorax coloration shifted more strongly towards orange in females originating from MB populations (change $-24.16 \pm 3.89 \mathrm{SE}$ ) than in adult females originating from FB population (change $-4.27 \pm 1.50 \mathrm{SE}$, Fig. 3 ).

\section{Discussion}

A recent study suggested that female common lizards exhibit distinct genetically determined colour morphs (Vercken et al., 2007). Here, we analysed the frequency distribution of thoracic colour parameters (hue, chroma and brightness) in female common lizards. We compared colour parameters among the three visual colour classes

Table 1 Effects of the adult sex ratio (ASR) manipulation on repeated measurements of body coloration on the thorax of adult female common lizards during ( $n=62$ females) and after the ASR manipulation ( $n=24$ females).

\begin{tabular}{|c|c|c|c|c|}
\hline Factors & & Chroma & Hue & Brightness \\
\hline \multicolumn{5}{|c|}{ During the ASR manipulation } \\
\hline Time & Test statistics & $F_{1,60}=16.54, P=0.001$ & $F_{1,60}=2.16, P=0.14$ & $F_{1,60}=19.59, P<0.001$ \\
\hline ASR & Test statistics & $F_{1,60}=0.20, P=0.66$ & $F_{1,60}=0.032, P=0.86$ & $F_{1,60}=0.115, P=0.74$ \\
\hline Time $\times$ ASR & Test statistics & $F_{1,60}=3.08, P=0.09$ & $F_{1,60}=11.52, P=0.001$ & $F_{1,60}=2.89, P=0.09$ \\
\hline \multirow[t]{2}{*}{ Female identity } & Test statistics & $\chi^{2}=44.27, P<0.0001$ & $\chi^{2}=22.74, P<0.0001$ & $\chi^{2}=5.40, P=0.02$ \\
\hline & ICC & 0.72 & 0.55 & 0.29 \\
\hline \multicolumn{5}{|c|}{ After the ASR manipulation } \\
\hline Time & Test statistics & $F_{1,21}=2.88, P=0.10$ & $F_{1,21}=8.09, P=0.01$ & $F_{1,21}=29.91, P<0.0001$ \\
\hline ASR & Test statistics & $F_{1,21}=4.51, P=0.046$ & $F_{1,21}=12.51, P=0.002$ & $F_{1,21}=0.58, P=0.46$ \\
\hline Time $\times$ ASR & Test statistics & $F_{1,21}=2.46, P=0.13$ & $F_{1,21}=22.84, P=0 . .0001$ & $F_{1,21}=0.003, P=0.96$ \\
\hline \multirow[t]{2}{*}{ Female identity } & Test statistics & $\chi^{2}=3.85, P=0.049$ & $\chi^{2}=16.86, P<0.0001$ & $\chi^{2}<0.01, P>0.92$ \\
\hline & ICC & 0.37 & 0.72 & $<0.001$ \\
\hline
\end{tabular}

Results were obtained with a mixed-effect model that accounted for random variation among females. Intra-class correlation coefficients (ICC) were calculated as from the variance components of the model as the ratio of the variance between females on the sum of the variance between and within females (residual variance). 


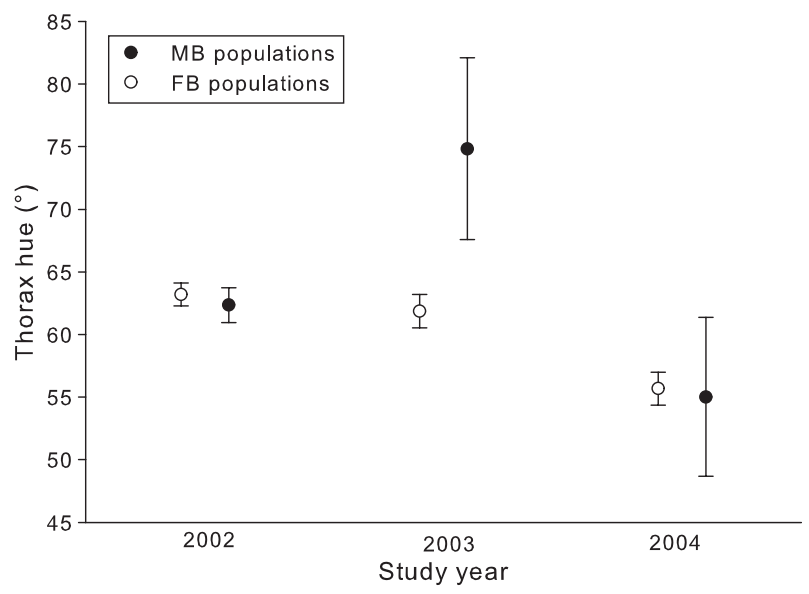

Fig. 3 Ventral colouration (thorax hue) of adult female common lizards, Lacerta vivipara, during and after the adult sex ratio (ASR) manipulation. Hue (mean and standard errors) was calculated for each female in each treatment before the ASR manipulation (2002), at the end of the ASR manipulation (2003) and 1 year after the ASR manipulation (2004). Hue is an angular measurement of the redness of the ventral coloration where larger values indicate a more pale yellow coloration. Hue did not differ between ASR treatments in $2002\left(F_{1,10}=0.213, P=0.65\right)$ and $2004\left(F_{1,6}=0.03, P=0.87\right)$, but females from $\mathrm{MB}$ populations were more pale yellow than females from $\mathrm{FB}$ populations in $2003\left(F_{1,7}=5.87, P=0.04\right)$. See text and Table 1 for a detailed analysis of intra-individual changes in colouration.

(pale yellow, orange and intermediate) previously described by Vercken et al. (2007) and investigated the existence of clusters of individuals with distinct colour parameters. As expected, visual colour classes differed in their colour parameters. However, our analyses of reflectance spectra and whole-body digital pictures could not corroborate the existence of discrete colour classes. First, spectral data were distributed continuously following a normal distribution, as for colour data from digital photos including larger areas than spectrophotometer measurements (see Supplementary material). Second, the mixture models did not reveal consistent clusters of individuals. We found two clusters of individuals in one analysis including hue and chroma, but these two clusters were not found in other analyses and in the data set of Vercken et al. (2007). Furthermore, these two clusters did not discriminate well the pale yellow females from the two other visual colour classes. Similarly, Vercken et al. (2007) used a discriminant analysis to separate the three colour classes. Their analysis did not well separate intermediate females from the other two classes and, here, we found no evidence for clusters in their data. Taken together, these results show that putative morphs cannot be easily distinguished based solely on visual parameters (human vision and spectral data). Endler and Mielke (2005) recently proposed a threshold similarity between two colour patterns below which they cannot be discriminated. As reflectance spectra strongly overlapped between visual colour classes of females, this threshold is probably not attained in this species. Third, a quantitative analysis of colour heterogeneity over the entire ventral area, among scales and within scales revealed that mixed coloration patterns were rare and that coloration was not more variable in intermediate females than in pale yellow or orange females. This last result questions the view that intermediate females have a mixed yellow and orange coloration arising from the joint expression of alleles for yellow and orange pigments (Vercken et al., 2007).

We assessed some of the determinants of variation in thorax colouration by: (1) examining the relationship between colouration and morphological traits; (2) testing for plasticity in colouration in response to male aggression; and (3) measuring the inter-annual consistency of the colouration. Our analyses support the existence of both fixed and condition-dependent components of ventral coloration. Before the sex ratio manipulation, body size explained part of the variation in colour traits: larger females were redder and had purer ventral colours than smaller females. Larger individuals are older individuals, often dominate social interactions and have a higher reproductive success, and older females use different mating tactics than younger females (Massot et al., 1992; Richard et al., 2005). This size-dependent variation may thus reflect differences in social status, reproductive strategies or age between females (Martin $\delta$ Forsman, 1999; Weiss, 2006) and may therefore be used as signal for competitors.

In addition, we found strong plastic changes in the redness of the ventral coloration, but not in purity and brightness. One year after the start of the manipulation, females of the male-biased ASR showed substantially decreased redness (Fig. 3). The change induced by the ASR manipulation (contrast between treatments $13.4^{\circ}$ ) was similar to the difference observed between orange and pale yellow females before the manipulation (contrast between visual colour classes $14.5^{\circ}$ ). Previously, we reported that females were subjected to increased mating attempts by males in male-biased populations, and that male-biased ASR decreased female survival and reproduction, but did not cause obvious changes in inter-sexual competition (Fitze et al., 2005; Le Galliard et al., 2005). Here, we further demonstrate that male aggression can influence carotenoid-based coloration in females.

This effect of male aggression on coloration in fully grown adult females might be due to two nonexclusive mechanisms. First, male harassment during mating may reduce the capacity of females to acquire carotenoids in their skin tissues. Carotenoids cannot be synthesized de novo, the availability of carotenoids is limited in nature, and the quantity of carotenoids ingested is determined by diet quantity and composition (Olson $\delta$ Owens, 1998). Male harassment during mating may 
cause suboptimal foraging and limit the ability of females to acquire carotenoids in their diet. In several fish species, harassment by males decreases feeding opportunities for females (Magurran \& Seghers, 1994; Schlupp et al., 2001). It is usually speculated that this should negatively affect fecundity for females (Magurran \& Seghers, 1994); thus, our study suggests that a potential cost of male harassment for females may be limited intake of carotenoids. On the other hand, enhanced male mating attempts can be physiologically stressful and can induce considerable changes in females' physiology. For example, male aggression caused severe wounds in females (Le Galliard et al., 2005), which might increase infection by pathogens and stress. Both stress and immunological challenge are predicted to divert carotenoids used for colouration through increased oxidative stress (Olson \& Owens, 1998). Thus, in response to enhanced male aggression, carotenoid-based ventral coloration might have been traded-off with carotenoid-based defence to oxidative stress.

The function and the origin of carotenoid-based coloration have, however, been poorly studied in common lizard. Unpublished data showed that the carotenoid content of the skin is well correlated with the hue of the lizard's skin coloration, explaining around $47 \%$ of the observed variation (Cote, 2006; P.S. Fitze, J. Cote, C. Isaksson, S. Andersson, J.-M. Rossi \& J. Clobert, unpublished results). This finding shows that skin colour intensity is importantly influenced by skin carotenoid content. Surprisingly, however, carotenoid supplementation in the diet had no effect on ventral coloration in this species, whereas a chronic stress response through corticosterone supplementation positively affected the ventral coloration in adults. Thus, carotenoid acquisition might not be the main proximate mechanism involved in ventral coloration, suggesting that other, yet unknown, mechanisms may determine the link between ventral coloration and an animal's capacity to cope with stress (Cote, 2006, P.S. Fitze et al., unpublished results).

After the ASR manipulation, we released all females into populations with a female-biased ASR (i.e. mean natural sex ratio). One year later, females from malebiased populations had shifted back their ventral coloration towards orange and had fully compensated for the past effect of male aggression (Fig. 3). Our study involved repeated measures conducted on the same females. This result thus provides evidence for reversible plasticity, and thus flexibility in the ventral colouration of adult females. Despite ASR-induced plasticity in ventral coloration for hue, there was no significant change in purity and brightness of ventral coloration and there was significant individual consistency of the colour parameters between years (intra-class correlation: $r \approx 0.3-0.7$ ). These results clearly imply that different colour-related traits may display different sensitivity to environmental factors. Moreover, the inter-year consistency described here is in agreement with the significant correlation between mother's and daughter's colourations found by Vercken et al. (2007), which provided an estimate of broad-sense heritability of 0.48 or 0.62 with or without mixed females respectively. This indicates that a significant portion of the phenotypic variation in the ventral colouration of adult females may be due to genetic effects, maternal effects or long-lasting effects of early environmental conditions. Maternal and post-natal effects are good candidates in the common lizard, as several studies have shown that conditions experienced by mothers during pregnancy and by juveniles early in life can have long-lasting effects on offspring life-history traits (Le Galliard et al., 2006). However, additional manipulative studies, breeding designs and pedigree analyses will be needed to disentangle genetic components of colour variation in adult female common lizards from environmental variation.

\section{Acknowledgments}

We thank E. Svensson, M. Jennions and J. Clobert and one anonymous reviewer for comments on an earlier version, as well as D. Mersch and M.-L. Jarzat for field assistance. Funds were received from the French Ministry of Education and Research (ACI 'Jeunes Chercheurs 2001'), the French Ministry of Environment (ACI 'Invasions biologiques'), the European Research Training Network 'ModLife' (HPRN-CT-2000-00051), the Federal Office of Education and Science (BBW No. 01.0254 to P.S.F), the Swiss National Science Foundation (No. PBBEA-104428 to P.S.F), and the Spanish Ministry of Education and Science (CGL2005-01187).

\section{References}

Bauwens, D. 1987. Sex recognition by males of the lizard Lacerta vivipara: an introductory study. Amphib.-Reptil.. 8: 49-57.

Cooper, W.E. \& Greenberg, N. 1992. Reptilian coloration and behavior. In: Biology of the Reptilia, Vol. 18 (C. Gans \& D. Crews, eds), pp. 298-422. University Chicago Press, Chicago, IL.

Cote, J. 2006. Socially acquired information in the common lizard (Lacerta vivipara). Ecology. Universite Pierre et Marie Curie, Paris.

Endler, J.A. 1990. On the measurement and classification of colour in studies of animal colour patterns. Biol. J. Linn. Soc. 41: 315-352.

Endler, J.A. 1991. Variation in the appearance of guppy colour patterns to guppies and their predators under different visual conditions. Vision Res. 31: 587-608.

Endler, J.A. \& Mielke, P.W. 2005. Comparing entire colour patterns as birds see them. Biol. J. Linn. Soc. 86: 405-431.

Fitze, P.S., Kolliker, M. \& Richner, H. 2003. Effects of common origin and common environment on nestling plumage coloration in the great tit (Parus major). Evolution 57: 144-150.

Fitze, P.S., Le Galliard, J.-F., Federici, P., Richard, M. \& Clobert, J. 2005. Conflict over multiple partner mating among males and females of polygynandrous common lizards. Evolution 59: 2451-2459. 
Fraley, C. \& Raftery, A.E. 1999. Software for model-based cluster analysis. J. Classification 16: 297-306.

Galeotti, P., Rubolini, D., Dunn, P.O. \& Fasola, M. 2003. Colour polymorphism in birds: causes and functions. J. Evol. Biol. 16: 635-646.

Hill, G.E. \& Montgomerie, R. 1994. Plumage colour signals nutritional condition in the house finch. Proc. R. Soc. B: Biol. Sci. 258: 47-52.

Hoffman, E.A. \& Blouin, M.S. 2000. A review of colour and pattern polymorphisms in anurans. Biol. J. Linn. Soc. 70: 633665.

Le Galliard, J.-F., Fitze, P.S., Ferriere, R. \& Clobert, J. 2005. Sex ratio bias, male aggression, and population collapse in lizards. Proc. Natl Acad. Sci. U. S. A. 102: 18231-18236.

Le Galliard, J.F., Massot, M., Landys, M.M., Meylan, S. \& Clobert, J. 2006. Ontogenic sources of variation in sexual size dimorphism in a viviparous lizard. J. Evol. Biol. 19: 690-704.

LeBas, N.R. \& Marshall, J. 2000. The role of colour in signalling and male choice in the agamid lizard Ctenophorus ornatus. Proc. R. Soc. B Biol. Sci. 267: 445-452.

Magurran, A.E. \& Seghers, B.H. 1994. A cost of sexual harassment in the guppy, Poecilia reticulata. Proc. R. Soc. B Biol. Sci. 258: 89-92.

Martin, J. \& Forsman, A. 1999. Social costs and development of nuptial coloration in male Psammodromus algirus lizards: an experiment. Behav. Ecol. 10: 396-400.

Massot, M., Clobert, J., Pilorge, T., Lecomte, J. \& Barbault, R. 1992. Density dependence in the common lizard: demographic consequences of a density manipulation. Ecology $\mathbf{7 3}$ : 1742-1756.

Milinski, M. \& Bakker, T.C.M. 1990. Female sticklebacks use male coloration in mate choice and hence avoid parasitized males. Nature 344: 330-333.

Møller, A.P., Biard, C., Blount, J.D., Houston, D.C., Ninni, P., Saino, N. \& Surai, P.F. 2000. Carotenoid-dependent signals: indicators of foraging efficiency, immunocompetence or detoxification ability? Avian Poult. Biol. Rev. 11: 137-159.

Olson, V.A. \& Owens, I.P.F. 1998. Costly sexual signals: are carotenoids rare, risky or required? Trends Ecol. Evol. 13: 510514.

Pinheiro, J.C. \& Bates, D.M. 2000. Mixed-Effects Models in $S$ and $S$-PLUS. Springer-Verlag, New York.

Richard, M., Lecomte, J., De Fraipont, M. \& Clobert, J. 2005. Age-specific mating strategies and reproductive senescence. Mol. Ecol. 14: 3147-3155.

Roff, D.A. 1996. The evolution of threshold traits in animals. Q. Rev. Biol. 71: 3-35.

Roulin, A. 2004. The evolution, maintenance and adaptive function of genetic colour polymorphism in birds. Biol. Rev. 79: $815-48$.
Schlupp, I., McKnab, R. \& Ryan, M.J. 2001. Sexual harassment as a cost for molly females: bigger males cost less. Behaviour 138: $277-286$.

Sinervo, B. \& Calsbeek, R. 2006. The developmental, physiological, neural, and genetical causes and consequences of frequency-dependent selection in the wild. Annu. Rev. Ecol. Evol. Syst. 37: 581-610.

Sinervo, B. \& Lively, C.M. 1996. The rock-paper-scissors game and the evolution of alternative male strategies. Nature $\mathbf{3 8 0}$ : 240-243.

Thompson, C.W., Moore, I.T. \& Moore, M.C. 1993. Social, environmental and genetic factors in the ontogeny of phenotypic differentiation in a lizard with alternative male reproductive strategies. Behav. Ecol. Sociobiol. 33: 137-146.

Venable, W.N. \& Ripley, B.D. 1999. Modern Applied Statistics with $S$-PLUS. Springer-Verlag, New York.

Vercken, E. \& Clobert, J. 2008. Ventral color polymorphism correlates with alternative behavioral patterns in female common lizards (Lacerta vivipara). Ecoscience 15: in press.

Vercken, E., Massot, M., Sinervo, B. \& Clobert, J. 2007. Colour variation and alternative reproductive strategies in females of the common lizard Lacerta vivipara. J. Evol. Biol. 20: 221232.

Weiss, S.L. 2006. Female-specific color is a signal of quality in the striped plateau lizard (Sceloporus virgatus). Behav. Ecol. 17: $726-732$.

Received 10 January 2008; revised 7 March 2008; accepted 11 March 2008

\section{Supplementary material}

The following supplementary material is available for this article:

Table S1 Proportion of homogeneous, slightly heterogeneous and heterogeneous scales for ventral coloration in female common lizards $(n=86)$.

This material is available as part of the online article from: http://www.blackwell-synergy.com/doi/abs/10.1111/ j.1420-9101.2008.01534.x

Please note: Blackwell Publishing are not responsible for the content or functionality of any supplementary materials supplied by the authors. Any queries (other than missing material) should be directed to the corresponding author for the article. 